\title{
Evaluation of the Incomplete Gamma Function of Imaginary Argument by Chebyshev Polynomials
}

\section{By Richard Barakat}

During the course of some work on the diffraction theory of aberrations it was necessary to evaluate numerically the incomplete gamma function of imaginary argument $\gamma(\nu, i x)$ for certain values of the parameter $\nu$. These integrals are special cases of the confluent hypergeometric function, and in the standard notation [1]

$$
\begin{aligned}
\gamma(\nu, i x) & =\int_{0}^{i x} e^{-t} t^{\nu-1} d t & \operatorname{Re}(\nu)>0 \\
& =(i x)^{\nu} \nu^{-1}{ }_{1} F_{1}(\nu, 1+\nu,-i x) &
\end{aligned}
$$

where ${ }_{1} F_{1}$ is the confluent hypergeometric function. Only the case of $\nu$ real is considered.

When $v$ is an integer, ${ }_{1} F_{1}$ is simply a polynomial in $x$. It is also possible to evaluate (1) in terms of the Fresnel integrals when $(\nu-1)$ is a half-integer. For $\nu=\frac{1}{2},(1)$ is proportional to the Fresnel integral. For other half-integer values, we can use the recurrence relation

$$
\gamma(1+\nu, i x)=\nu \gamma(\nu, i x)-(i x)^{\nu} e^{-i x}
$$

to generate the necessary formulas. An alternate procedure utilizes the Lommel functions of two variables [2]; unfortunately, the Lommel functions have not been extensively tabulated.

The remaining values of $\nu$ can be treated by a Taylor series expansion for small values of $x$ and by asymptotic developments for large values of $x$. The main difficulty is the intermediate range where $x$ is neither large nor small. A possible method is to use Nielsen's [3] representation

$$
\gamma(\nu, i x+i y)-\gamma(\nu, i x)=e^{-i x}(i x)^{\nu-1} \sum_{n=0}^{\infty}(1-\nu)_{n}(-i x)^{-n}\left[1-e^{-i y} e_{n}(i y)\right]
$$

where $e_{n}(i y)$ is the truncated exponential series

$$
e_{n}(i y)=\sum_{m=0}^{n} \frac{(i y)^{m}}{m !}
$$

This method seems unnecessarily complicated.

A more powerful method is to utilize the Chebyshev polynomials. Instead of expanding the exponential in (1) into a Taylor series, we expand into a series of Chebyshev polynomials. As Lanczos [4] has pointed out, "While the expansion into powers on the basis of the Taylor's series gives the slowest convergence, the expansion into the Chebyshev polynomials gives the fastest convergence."

It is convenient to transform the integral slightly by setting $t=i x q$ in. (1)

Received March 4, 1960. 


$$
\gamma(\nu, i x)=(i q)^{\nu} \int_{0}^{1} e^{-i x q} q^{\gamma-1} d q
$$

Expanding the exponential into the series [5]

$$
e^{-i x q}=\sum_{n=0}^{\infty} \epsilon_{n}(-i)^{n} J_{n}(x) T_{n}(q) \quad|q| \leqq 1
$$

where $\epsilon_{n}$ is Newmann's factor $\left(\epsilon_{0}=2 ; \epsilon_{n}=1\right.$ for $n \geqq 1$ ) and $T_{n}(q)$ is the Chebyshev polynomial of the first kind

$$
\begin{aligned}
& T_{0}(q)=1 \\
& T_{n}(q)=\frac{n}{2} \sum_{s=0}^{[n / 2]} \frac{(-1)^{s}(n-s-1) !}{s !(n-2 s) !}(2 q)^{n-2 s} .
\end{aligned}
$$

Substituting (6) into (5) and interchanging sum and integral (this is permissible because of uniform convergence of the series) leads to the integrals

$$
\begin{aligned}
B_{0}(\nu) & \equiv \frac{1}{\nu} \\
B_{n}(\nu) & \equiv \int_{0}^{1} T_{n}^{\prime}(q) q^{\nu-1} d q \\
& =\frac{n}{2} \sum_{s=0}^{[n / 2]} \frac{(-1)^{s}(n-s-1) ! 2^{n-2 s}}{s !(n-2 s) !(n-2 s+\nu)} .
\end{aligned}
$$

Finally (5) hecomes

$$
\gamma(\nu, i x)=(i \varphi)^{\nu} \sum_{n=0}^{\infty} \epsilon_{n}(-i)^{n} J_{n}(x) B_{n}(\nu) .
$$

To obtain an error estimate on $B_{n}$ for large $n$, we use the relation

$$
T^{\prime}{ }_{n}(q)=\cos n \theta, \quad q=\cos \theta
$$

so that (8) becomes

$$
\begin{aligned}
B_{n}(\nu) & =\int_{0}^{\pi / 2} \cos n \theta(\cos \theta)^{\nu-1} \sin \theta d \theta \\
& \sim 0(1) .
\end{aligned}
$$

The asymptotic expression for $J_{n}(x)$ when $n$ is large and $x$ is fixed is given by [6]

$$
J_{n}(x) \sim \frac{(x / 2)^{n}}{n !} \quad\left(\frac{x^{2}}{4} \ll n\right) .
$$

Hence the dominating factor is $J_{n}(x)$.

As an example of the power of the method, we consider the case of the Fresnel integrals $\left(\nu=\frac{1}{2}\right)$, using as a standard the recent table by Pearcey [7]. The first thirteen values of $B_{n}(.5)$ are:

$$
\begin{aligned}
& B_{0}=2.0 \\
& B_{1}=0.66666667 \\
& B_{2}=-1.20 \\
& B_{3}=-0.85714286 \\
& B_{4}=0.57777778 \\
& B_{5}=0.52813853 \\
& B_{6}=-0.54358974
\end{aligned}
$$

$$
\begin{aligned}
& B_{7}=-0.49696970 \\
& B_{8}=0.42976370 \\
& B_{9}=0.40710869 \\
& B_{10}=-0.40752711 \\
& B_{11}=-0.38709818 \\
& B_{12}=0.35566473
\end{aligned}
$$


Omitting the superfluous factor $(i q)^{.5}$ in (5) yields

$$
\begin{aligned}
\gamma_{1}(.5, i x) & =\gamma(.5, i x)(i q)^{-.5} \\
& =\sum_{n=0}^{\infty} \epsilon_{n}(-i)^{n} J_{n}(x) B_{n}(.5) \\
& =\left(\frac{2 \pi}{x}\right)^{1 / 2}[C(x)-i S(x)] .
\end{aligned}
$$

\begin{tabular}{|c|c|c|c|c|}
\hline \multirow{4}{*}{$\begin{array}{l}\text { Pearcey } \\
\text { series }\end{array}$} & \multicolumn{3}{|c|}{$\operatorname{Re}\left[\gamma_{1}(.5, i x)\right]$} & \\
\hline & $\begin{array}{r}x=1 \\
1.8090490\end{array}$ & $\begin{array}{r}x=2 \\
1.335193\end{array}$ & $\begin{array}{r}x=3 \\
0.811910\end{array}$ & \\
\hline & 1.80904 & $1.3351931 \quad(5)$ & 0.8119099 (6) & $0.461+622(\tau)$ \\
\hline & 1.8090 & $1.3351744(4)$ & 0.8118993 & $0.4614576(6)$. \\
\hline
\end{tabular}

The results for various values of $x$ are tabulated below. The numbers in parentheses indicate the number of terms used in the series expansion.

Similar results hold for the imaginary part of $\gamma_{1}$. The small number of terms required for six-place accuracy is remarkable compared with the usual Taylor series expansion.

Using this method, integrals have been computed corresponding to $\nu=.2, .4$, $.6, .8$ in the interval $0(.1) 5$. The integrals in question are

$$
\begin{aligned}
\gamma_{1}(\nu, i x) & =\int_{0}^{1} e^{-i x q} q^{\nu-1} d q \\
& =(i q)^{-\nu} \gamma(\nu, i x)
\end{aligned}
$$

\begin{tabular}{|c|c|c|c|c|}
\hline$B_{\mathrm{n}} / \nu$ & .2 & .4 & .6 & .8 \\
\hline$B_{0}$ & 5.00000000 & 2.50000000 & 1.66666667 & 1.25000000 \\
\hline$B_{1}$ & .83333333 & .71428571 & .62500000 & .555555 .56 \\
\hline$B_{2}$ & -4.09090909 & -1.66666667 & $-.897+3590$ & $-.53571+29$ \\
\hline$B_{3}$ & -1.25000000 & -.96638655 & -.76388889 & $-.61+03509$ \\
\hline$B_{4}$ & 3.26839827 & $.98+84848$ & $.3288740: 3$ & .05952381 \\
\hline$B_{5}$ & .99358973 & .65203859 & .42658730 & .27324056 \\
\hline$B_{6}$ & -3.08546292 & -.90909091 & -.32988751 & $-.1155+62 ;$ \\
\hline$B_{7}$ & -.98290598 & -.62150387 & -.39839182 & $-.257 \cdot 26340$ \\
\hline$B_{8}$ & 2.86921706 & .76839826 & $.237+2520$ & .05315763 \\
\hline$B_{9}$ & .90301003 & .53065850 & .31171679 & $.18010633 !)$ \\
\hline$B_{10}$ & -2.76714376 & -.72594072 & -.23077198 & $-.067+4618$ \\
\hline$B_{11}$ & -.88701757 & -.50877478 & -.29504438 & $-.17180+11$ \\
\hline$B_{12}$ & 2.65095170 & .65829331 & .19072285 & $.0425+734$ \\
\hline$B_{13}$ & .8439206 & .4620702 & .2532754 & .1368987 \\
\hline$B_{14}$ & -2.581791 & -.630248 & -.185156 & -.048825 \\
\hline$B_{15}$ & $-.62+846$ & -.335736 & -.187154 & -.108511 \\
\hline
\end{tabular}

(see equation 5).

The first fifteen values of $B_{n}(\nu)$ are listed in Table 1 , while in Table 2 we give the values of the real and imaginary values of $\gamma_{1}(\nu, i x)$ to six decimals.

TABLE 1 
Table 2

\begin{tabular}{|c|c|c|c|c|c|c|c|c|c|}
\hline \multicolumn{5}{|c|}{$\operatorname{Re}\left[\gamma_{1}(\nu, i x)\right]$} & \multicolumn{5}{|c|}{$\operatorname{Im}\left[\gamma_{1}(\nu, i x)\right]$} \\
\hline$x / \nu$ & .2 & .4 & .6 & .8 & $x / y$ & .2 & .4 & .6 & .8 \\
\hline 0 & & & 1.6666567 & 1.250000 & 0 & 0 & 0 & 0 & 0 \\
\hline .1 & & & $1.66 j 4744$ & 1.248 & & -.083281 & -.071380 & -.062454 & -.055512 \\
\hline .2 & & & 1. 658989 & 1.242871 & .2 & -.166251 & -.142465 & $-.124630 \mid$ & -.110761 \\
\hline .3 & & & 1. 649432 & 1. 233999 & .3 & -.248598 & -.212966 & -.186254 & -.165486 \\
\hline .4 & 4.963889 & $2.46(j)(0)$ & 1.636128 & $1.2216 ; 50$ & .4 & -.330016 & -.282593 & -.247052 & -.219430 \\
\hline .5 & & & 1. 619153 & 1.205896 & .5 & -.410206 & -.351063 & -.306759 & -.272340 \\
\hline .6 & & & 1.598600 & 1.18 & .6 & -.488874 & -.418102 & -.365115 & -.323971 \\
\hline .7 & & & $1.57458 f ;$ & 1.164560 & .7 & -.565736 & -.483443 & -.421868 & -.374084 \\
\hline .8 & & 2.3 & 1.547245 & 1.139217 & .8 & -.640519 & -.546831 & -.476778 & -.422454 \\
\hline .9 & & & 16730 & 1.110945 & .9 & -.712964 & -.608020 & -.529616 & -.468863 \\
\hline 1.0 & & & 3.3209 & 1.0 & 1.0 & -.782825 & -.66 & -.58 & -.51 \\
\hline 1.1 & & & 1.446869 & 1.046282 & 1.1 & -.849872 & -.722903 & -.628226 & -.554999 \\
\hline 1.2 & & & 1.407910 & 1.010259 & 1.2 & -.913890 & -.776182 & -.673611 & -.594363 \\
\hline 1.3 & & $39) 39$ & 1.36 fis.45 & .972044 & $1: 3$ & -.974687 & -.826440 & -.716151 & -.631041 \\
\hline 1.4 & & 21 & 1.322998 & .93 & 1.4 & -1.032085 & -.87 & -.75 & -.66 \\
\hline 1.5 & & & 1.277506 & .889904 & 1.5 & -1.085929 & -.917259 & -.792115 & -.695794 \\
\hline 1.6 & & & 1.230312 & .846438 & 1.6 & -1.136085 & -.957555 & -.825293 & -.723642 \\
\hline 1.7 & & & $16 f f 7$ & (j91) & 1.7 & -1.18 & -.994298 & -.855137 & -.7 \\
\hline 1.8 & & & 1828 & 908 & 1.8 & -1.224902 & -1.0 & -.88 & -.7 \\
\hline 1.9 & 4.2 & & 1.081054 & .709337 & 1.9 & -1.263406 & -1.056824 & -.904557 & -.788113 \\
\hline 2.0 & 4.2 & 1. 805022 & 1.02:)(6)8 & (iki;2227 & 2.0 & -1.297904 & -1.082511 & -.924050 & -.803095 \\
\hline 2.1 & 4.1 & 787!) & 7750 & 827 & 2.1 & -1.32 & -1 & -.9 & -.81 \\
\hline 2.2 & & i. $690+47$ & .025742 & 385 & 2.2 & -1.35 & -1.12 & -.95 & -.8 \\
\hline 2.3 & & 1.(6i) & .873840 & 143 & 2.3 & -1.377251 & -1.137140 & -.961600 & -.828439 \\
\hline 2.4 & 3.9 & 1.5757!)8 & $.8222 !) 6$ & $.4733+2$ & 2.4 & -1.395725 & -1.14 & -.96 & -.830468 \\
\hline 2.5 & & 107 & .7713 .56 & 21.4 & 2.5 & -1.4 & -1 & -.9 & -.8 \\
\hline 2.6 & & 177 & .721257 & & 2.6 & -1.42 & -1.1 & -.9 & -.82 \\
\hline 2.7 & & 3251 &.$(j 7: 227$ & & 2.7 & -1.428133 & -1.159223 & -.964540 & -.81 \\
\hline 2.8 & 3.7306 & i. 3551558 &.$(i 2.4833$ & .295()5!) & 2.8 & -1.431615 & -1.156262 & -.957449 & -.80 \\
\hline 2.9 & & & 28 & & & -1.4 & -1. & -.9 & -.7 \\
\hline 3.0 & & $7: 35$ & $.5333(i .55$ & & 3.0 & -1.42 & -1.1 & -.9 & -.78 \\
\hline 3.1 & & $(x)$ & $4(9)(9) 3 !$ & $.17(i 3394$ & 3.1 & -1.422082 & -1.129203 & -.91 & -.76 \\
\hline 3.2 & & ;285 & $.+502+11$ & $.140(335$ & 3.2 & -1.412821 & -1.114693 & -.90 & -.74 \\
\hline 3.3 & & 1.1 &.+1 & $(010$ & 3.3 & -1.40 & -1.0 & -.8 & -.7 \\
\hline 3.4 & & 9530 & $.375+4(i 2$ & .0756336 & 3. & $-1.38 t$ & -1 & $-.8 t$ & $-.6 \mathrm{~s}$ \\
\hline 3.5 & & $1.02(2) 75(0)$ & .341618 & & 3.5 & -1.369574 & -1.05 & -.83 & -.675167 \\
\hline 3.6 & & .992512 & $.3102(i 5$ & $.02(0) 18$ & 3.6 & -1.350732 & -1.03 & -.81 & -.649064 \\
\hline 3 & 3.2 & 78969 & $.281+77$ & -.00 & & -1.330 & -1 & -.7 & -.6 \\
\hline 3.8 & & .925076 & 308 & 0 & & -1.30 & -.8 & -.75 & $-.5 \mathrm{~s}$ \\
\hline 3.9 & 1871 & $.89(6787$ & .231794 & $-.04+625$ & 3.9 & $-1.28+170$ & -.957077 & -.729200 & -.564681 \\
\hline 4.0 & 8312 & .870358 & .210951 & -.061033 & 4.0 & -1.259431 & -.929226 & -.700045 & -.535242 \\
\hline & 7008 & $.8466(6) 6$ & .192780 & $-.07+871$ & & -1.233815 & -.900728 & -.67 & -.50 \\
\hline & 9754 & .825790 & .177259 & -.086163 & 4. & -1.2075 & -.871809 & -.64 & -.475667 \\
\hline 4.3 & 74730 & $.807(108$ & .164354 & -.094951 & 4. & -1.1808 & -.842691 & -.61 & -.445944 \\
\hline 4.4 & 3.052494 & .792104 & .154010 & -.101294 & 4. & -1.154033 & -.813590 & -.580990 & -.416526 \\
\hline & & .779212 & .146158 & -.105264 & & -1.127213 & -.784712 & & -.387604 \\
\hline & & .76885 & $.1+0713$ & & 4. & -1.100636 & -.756258 & -.52 & -.359357 \\
\hline 4.7 & 1852 & .760931 & .137576 & -.106457 & 4.7 & $-1.07+503$ & -.728417 & -.494873 & -.331955 \\
\hline 4.8 & 90025 & .755335 & .136634 & -.103895 & 4.8 & -1.049002 & -.701367 & -.467764 & -.305558 \\
\hline & & .751945 & .137763 & -.099392 & & & -.675274 & -.441722 & -.280310 \\
\hline & 2.973261 & .750625 & .140826 & -.093084 & & -1.000593 & -.650291 & -.416892 & -.256347 \\
\hline
\end{tabular}

The author wishes to thank Mrs. E. Lerin and Miss E. Kitrosser for help with the computations.

Research Laboratory

Itek Corporation

Boston, Massachusetts 
1. H. Bateman, Higher Transcendental Punctions, V. 2, MeGraw-Hill Book Co., Inc., New York, 1953.

2. G. N. Watson, Treatise on the Theory of Bessel Functions, Second Edition, Cambridge University Press, 1948, p. 544.

3. E. KREYszig, "On the Zeros of the Fresnel Integrals", Canad.J. Math., v. 9, 1957, p. 118 (also reference 1 ).

4. C. Lanczos, Tables of Chebyshev Polynomials $S_{n}(x)$ and $C_{n}(x)$, Nat. Bur. Standards, U. S. Government Printing Office, Washington D. C., 1952.

5. B. VAN DER POL \& T. J. WEIJERS, "Tchebycheff Polynomials and Their Relation to Circular Functions, Bessel Functions and Lissajous Figures," Physica, 1933.

6. N. W. McLachlan, Bessel Functions for Engineers, Second Edition, Oxford University Press, 1955, p. 86.

7. T. Prarcex, Table of the Fresnel Integral to Six Decimal Places, Cambridge University Press, 1956. 